\title{
1 Relating glutamate, conditioned, and clinical hallucinations via 1H-MR Spectroscopy
}

${ }^{1}$ Department of Psychiatry, Connecticut Mental Health Center, Yale University, New Haven, CT, 9 USA.

$10{ }^{2}$ Maryland Psychiatric Research Center, Department of Psychiatry, University of Maryland

11 School of Medicine, Catonsville, MD, USA.

$12{ }^{3}$ Department of Psychiatry, University of Chicago, Chicago, IL, USA.

14 *Corresponding Author: Philip Corlett; philip.corlett@yale.edu

1534 Park Street, New Haven, CT, 06511

16 Tel: 203-974-7866

Word Count: 150 (Abstract) +3867 (Abstract + text body) 


\section{ABSTRACT}

Background and Hypothesis: Hallucinations may be driven by an excessive influence of prior expectations on current experience. Initial work has supported that contention and implicated the anterior insula in the weighting of prior beliefs.

Study Design: Here we induce hallucinated tones by associating tones with the presentation of a visual cue. We find that people with schizophrenia who hear voices are more prone to the effect and using computational modeling we show they overweight their prior beliefs. In the same participants, we also measured glutamate levels in anterior insula, anterior cingulate, dorsolateral prefrontal and auditory cortices, using magnetic resonance spectroscopy.

Study Results: We found a negative relationship between prior-overweighting and glutamate levels in the insula that was not present for any of the other voxels or parameters.

Conclusions: Through computational psychiatry, we bridge a pathophysiological theory of psychosis (glutamate hypofunction) with a cognitive model of hallucinations (prioroverweighting) with implications for the development of new treatments for hallucinations.

Keywords: Conditioned hallucinations, Predictive Processing, Schizophrenia, Computational Psychiatry; MR Spectroscopy (MRS) 


\section{Introduction}

The predictive processing account of psychosis posits that hallucinations - percepts without an external stimulus - arise when prior beliefs are over-weighted relative to sensory evidence $^{1-3}$. If the incoming data are less precise (i.e. sensory uncertainty is overestimated), inference will conform to priors and hallucinations will result ${ }^{1}$. Participants who hallucinate tend to overweight prior beliefs in experimental tasks, e.g. they can be more readily conditioned to hallucinate an absent tone based on of previous learning that visual stimuli predict tones ${ }^{4-6}$. Here we replicate and extend this observation, testing the hypothesis that glutamate signaling underwrites the association between hallucinations and strong priors.

Predictive processing accounts posit a hierarchy of inference that is recapitulated in the anatomy and physiology of the brain ${ }^{7}$. Forward connections promulgate prediction errors up the hierarchy via AMPA receptor signaling? ${ }^{7}$. In contrast, backward connections mediate contextual effects - priors- downwards via NMDA receptors ${ }^{7}$. This division of labor is supported by primate data gathered from the visual system ${ }^{8}$. Blocking NMDA glutamate receptors - for example with the non-competitive NMDA receptor antagonist ketamine - induces a state redolent of endogenous psychosis ${ }^{9}$. It may do so by decreasing glutamatergic neurotransmission via NMDA receptors (hence the NMDA hypofunction model) but in addition, NMDA blockade on GABAergic interneurons increases glutamate release ${ }^{10}$ and thus, may increase transmission through AMPA receptors (hyper-glutamatergic accounts) ${ }^{11-13}$.

Proton magnetic resonance spectroscopy (MRS) studies in patients with schizophrenia (SCZ) suggest low glutamate levels ${ }^{14}$, although patients with more severe symptoms may have 
60

61

62

63

64

65

66

67

68

69

70

71

72

73

74

75

76

77

78

higher glutamate concentrations ${ }^{15-18}$. A follow-up study found elevated glutamate levels in left superior temporal gyrus but reduced glutamate in anterior cingulate cortex in hallucinators compared to non-hallucinators ${ }^{19}$. We aimed toward to clarify the relationship between hallucinations, psychosis, and glutamate using methods from computational psychiatry ${ }^{20-22}$.

We invited patients with SCZ, some of whom hallucinate and some of whom do not, as well as healthy control participants (CTR), to complete the conditioned hallucinations task ${ }^{4}$. On a separate occasion we measured glutamate levels in the auditory, anterior insular, anterior cingulate, and right dorsolateral prefrontal cortices. Previous work implicated left anterior insula in perceptual precision weighting and conditioned hallucinations: Powers and colleagues found that conditioned hallucinations engaged insula, while model-based parameters extracted from left insula were significantly greater in hallucinators, compared to non-hallucinators ${ }^{4}$. Based on this tripartite relation between insular activation, model parameters and hallucination status, we predicted that an alteration in glutamate levels in left anterior insula might play a role in endogenous psychosis and prior overweighting. The other three voxels were chosen based on their established link with other tasks used in the project (presented elsewhere) and were analyzed in a purely exploratory fashion.

\section{Methods}

\section{Participants}



demographics). They were recruited across two sites: the Connecticut Mental Health Center (CMHC), at Yale University and the Maryland Psychiatric Research Center (MPRC), at the University of Maryland School of Medicine. Participants were recruited via local advertisements or referred to the study through their clinician. Exclusion criteria included: 1 ) any neurological disorder or head trauma resulting in loss of consciousness or sustained deficits; 2) any recreational drug or alcohol use meeting criteria for at-risk drinking ( $>2$ drinks/day or 14 drinks/week for males, $>1$ drink/day or 7 drinks/week for females) for one month prior to enrollment or substance use disorder for 12 months prior to enrollment; 3) left-handedness; 4) self-reported abnormal hearing or vision; taking antipsychotic medication (Table 1). CTR had no family history of psychotic disorders, had not been previously diagnosed with a psychiatric disorder and had never been treated with psychotropic medications. All subjects gave written informed consent prior to the study, in accordance with the declaration of Helsinki. All procedures were approved by institutional review boards at Yale University and the University of Maryland School of Medicine.

\section{Clinical procedures}

97 Brief Psychiatric Rating Scale (BPRS) was used to assess symptom severity (Table 1). Item 12 of the BPRS was used to separate SCZ into hallucinators $\left(\mathrm{SCZH}^{+}\right)$and non-hallucinators $\left(\mathrm{SCZH}^{-}\right)$. 
100 had moderate-to-severe (BPRS-12=5) or severe (BPRS-12=6) hallucinations; the majority 101 experienced auditory hallucinations) and 17 non-hallucinators (Figure 1C). None of the CTR 102 endorsed any hallucinations (BPRS-12=1).

Table 1: Participant Characteristics (mean (SD))

\begin{tabular}{|c|c|c|c|c|c|}
\hline Variables & CTR $(n=24)$ & $S C Z H^{-}(n=17)$ & $\mathrm{SCZH}^{+}(\mathrm{n}=18)$ & Statistic & p value \\
\hline Age & $34.8(10.3)$ & $31.3(9.6)$ & $35.5(10.7)$ & $F=0.86$ & 0.43 \\
\hline Gender & $10 / 14$ & $11 / 6$ & $13 / 5$ & $\chi^{2}=4.42$ & 0.11 \\
\hline Race (African American/Caucasian/other/missing) & $3|17| 3 \mid 1$ & $8|9| 0$ & $4|12| 1 \mid 1$ & $\chi^{2}=8.94$ & 0.18 \\
\hline Participant Education & $15.8(1.9)$ & $14.1(1.2)$ & $14.5(1.6)$ & $F=6.42$ & 0.003 \\
\hline Maternal Education & $14.6(4.0)$ & $14.5(2.4)$ & $13.7(5.5)$ & $F=0.31$ & 0.73 \\
\hline Paternal Education & $14.5(4.6)$ & $14.0(4.7)$ & $14.0(6.9)$ & $F=0.05$ & 0.95 \\
\hline \multicolumn{6}{|l|}{ Neurocognitive test results } \\
\hline WTAR & $114.2(13.8)$ & $101.2(28.5)$ & $112.4(9.1)$ & $F=1.47$ & 0.39 \\
\hline MD Processing Speed & $55.2(14.0)$ & $42.5(14.4)$ & $45.2(16.2)$ & $F=3.31$ & 0.04 \\
\hline MD Working Memory & $55.8(7.9)$ & $43.1(15.2)$ & $46.4(16.6)$ & $F=4.03$ & 0.02 \\
\hline MD Verbal Learning & $49.2(6.3)$ & $42.9(14.5)$ & $42.3(15.7)$ & $F=1.81$ & 0.19 \\
\hline \multicolumn{6}{|l|}{ Clinical Ratings } \\
\hline BPRS Hallucinatory Behavior & $1.0(0.0)$ & $1.0(0.0)$ & $4.7(1.2)$ & - & - \\
\hline BPRS Delusions & $2.0(0.0)$ & $3.6(1.8)$ & $5.8(1.8)$ & $t=3.53^{1}$ & $0.001^{1}$ \\
\hline BPRS Negative Symptoms & $3.3(1.1)$ & $6.1(2.4)$ & $4.9(1.7)$ & $t=-1.72^{1}$ & $0.09^{1}$ \\
\hline Duration of Illness (yrs) & & $8.4(8.3)$ & $13.4(11.4)$ & $t=1.45$ & 0.16 \\
\hline \multicolumn{6}{|l|}{ Medication } \\
\hline Antipsychotic (Atypical |Typical) & & $16 \mid 1$ & $15 \mid 3$ & $\chi^{2}=1.00$ & 0.32 \\
\hline Antipsychotic medication: CPZ & alents (mg) & $278.4(259.5)$ & $388.47(208.98)$ & $t=0.58$ & 0.57 \\
\hline \multicolumn{6}{|l|}{ Other psychotropic medication } \\
\hline \multicolumn{6}{|c|}{ Antidepressant + Benzodiazepine + Mood } \\
\hline Antidepressant+ Benzodiazepi & & 0 & 3 & & \\
\hline Benzodiazepine & & 0 & 3 & & \\
\hline Antidepressant & & 4 & 4 & & \\
\hline Mood stabilizer & & 3 & 0 & & \\
\hline
\end{tabular}


WTAR=Wechsler Test of Adult Reading, measure of premorbid intelligence; MD Working Memory= Working memory domain (MATRICS); MD Processing Speed= processing speed domain (MATRICS); MD Verbal Learning=verbal learning domain (MATRICS); $\quad \mathrm{CPZ}=$ chlorpromazine equivalent; BPRS Delusions=Suspiciousness+Unusual Thought Content; BPRS Negative Symptoms=Emotional Withdrawal+Motor Retardation+Blunted Affect;

${ }^{1} \mathrm{SCZH}^{-}$vs. SCZH ${ }^{+}$

\section{Conditioned hallucinations task}

Participants were instructed to listen for a $1 \mathrm{kHz}$ tone, embedded in white noise and presented concurrently with a flashed visual stimulus (light- and dark-gray checkerboard) on a black background (Figure 1A). Stimulus presentation and data collection were controlled using Matlab 2017b (Mathworks, Natick, MA) and Psychtoolbox 3.0.12.

Threshold estimation was followed by 12 blocks of 30 trials. In each trial, a checkerboard flashed on the screen and a tone was played simultaneously at $25 \%, 50 \%$ or $75 \%$ detectionlikelihood tone intensity. Importantly, the tone was omitted on some trials (no-tone condition)

(Figure 1B, left). Trials were pseudorandomized within block. The likelihood of threshold tone presentation decreased non-linearly over blocks. The presentation of subthreshold and no-tone trials increased (Figure 1B, right). This distribution encouraged initial learning of audio-visual associations and then offered more opportunities for conditioned hallucinations $(\mathrm{CH})$ later in the experiment. Participants were instructed to press one response button if they heard a tone and another button to indicate that they heard no tone. They also held the response button down longer to indicate higher confidence in their choice. More information about the task and the stimuli can be found in Supplementary Methods. 


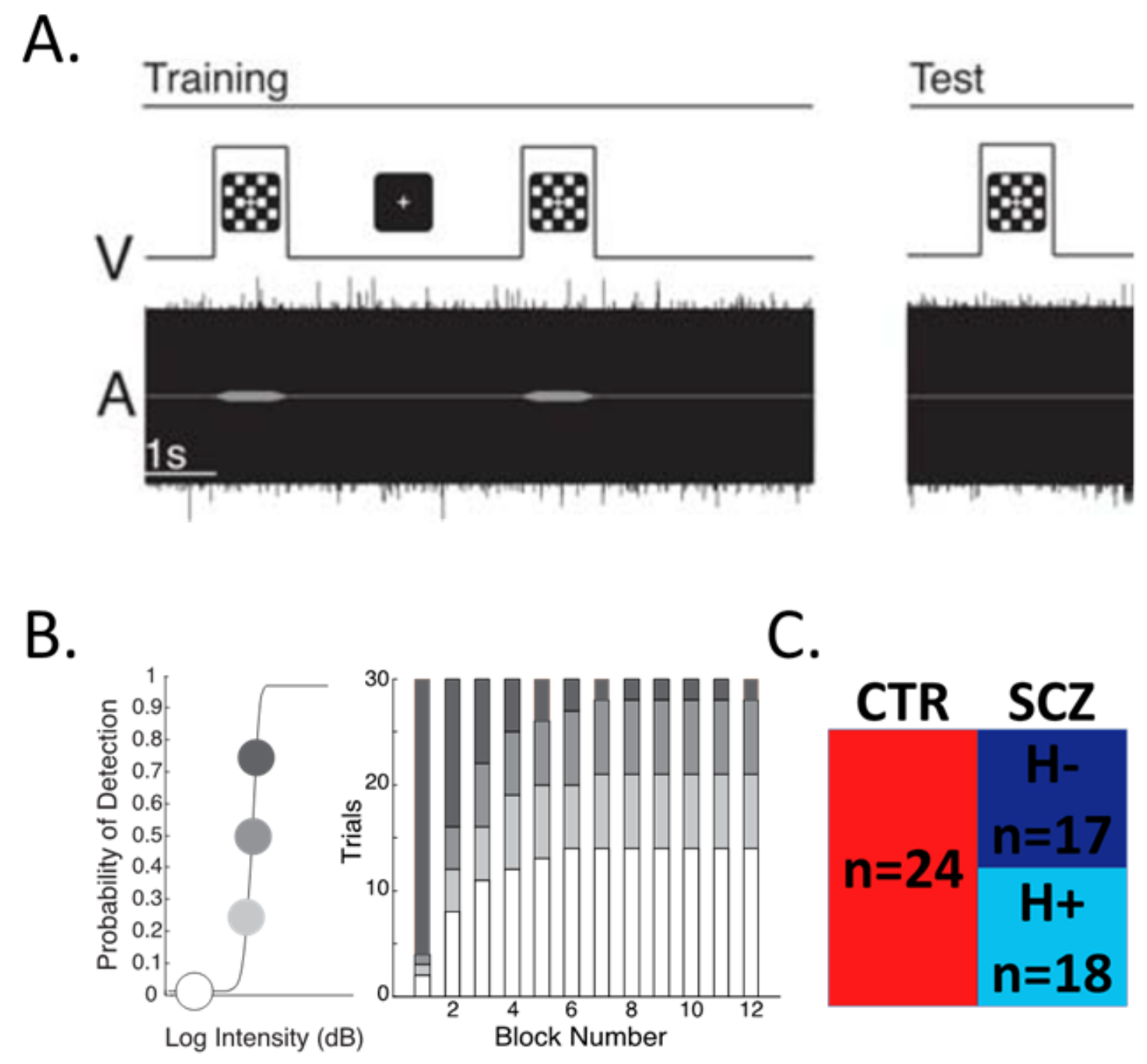

Figure 1: The conditioned hallucinations task. (A.) Participants worked to detect a tone embedded in

131 noise and presented concurrently with a visual checkerboard (B.) We estimated individual psychometric

132 curves for tone detection and varied tone intensity over 12 blocks of 30 trials (left). High-intensity tones

133 were more likely early and low-intensity and absent tones were more likely later (right). (C.) Our sample

134 consisted of 3, demographically-matched groups: 24 healthy controls (CTR; red), 17 schizophrenia- 
Glutamate, strong priors and hallucinations

135 patients without hallucinations $\left(\mathrm{SCZH}^{-}\right.$; dark blue) and 18 schizophrenia-patients with hallucinations $136\left(\mathrm{SCZH}^{+}\right.$; light blue).

137

\section{MRS data acquisition} different day) a 1H-MRS session (Table S1) at the Yale Magnetic Resonance Research Center or the University of Maryland Center for Brain Imaging Research. Participants were asked to stay awake and keep their eyes closed. Spectra were acquired from four voxels encompassing the anterior cingulate cortex (ACC), left anterior insula (LIN), right dorsolateral PFC (rdIPFC), and left auditory cortex (LAC) (see Figure 4A-D) We focused on "Glx" (Glutamate and Glutamine) which is reliably quantified with PRESS at $3 T$. More details about MRS can be found in the

\section{Supplementary Methods.}

\section{Statistical analysis} the number of and confidence in 'Yes' responses for trials where the tone was present (25\%, 50\%

151 and 75\%), as well as the confidence ratings when responding 'No'. No-response trials were 152 discarded from further analysis. All the analyses were performed using the Statistics toolbox of 153 MATLAB R2019b. Details about the statistical analysis can be found in the Supplementary 154 Methods. 


\section{Hierarchical Gaussian Filter}

(v.5.3.2) is freely available for download in the TAPAS package at

\section{https://translationalneuromodeling.github.io/tapas.}

The HGF is a generic hierarchical Bayesian model for inference in volatile environments.

167 volatility of those associations $\left(X_{3}\right)$ - how rapidly they change over time. Second-and third-level 
173

174

175

176

$177 \quad X_{\text {tone }}=p+\frac{1}{1+v}(s-p)$

178

where $s$ is the expected detection rate (depends on the trial-type: $0.25,0.5$ or 0.75 ) and $p$ is the

179

180

181

182

183

184

185

186

187

188

189

\section{Results}

\section{Sample characteristics}

prior expectation regarding the presence of a tone and relies on the audio-visual association that the subject has learnt so far (it is equal to $\hat{\mu}_{1}{ }^{23}$ ). Crucially, the relative contribution of prior $p$, compared to sensory input $s$, was controlled by parameter $v$ (an index of prior weighting, akin to Kalman gain ${ }^{25}$ ), and was specific to each subject. Based on the strong-prior theory of hallucinations, we expected higher $v$ values in the $\mathrm{SCZH}^{+}$group.

Eight variants of the HGF model were fitted to the behavioral data ('Yes'/'No' responses), each with different sets of free / fixed parameters (Table S3). The models were inverted at the subject-level, using trial-by-trial tone intensities and participant responses. The best-fitting model was detected through a Bayesian model comparison procedure based on the protected exceedance probability ${ }^{26}$. 
Glutamate, strong priors and hallucinations

192

193

194

195

196

197

198

199

200

201

202

203

204

205

206

207

208

209

Participant demographics are presented in Table 1. The groups were matched for age and sex, but there was a significant difference in years of education $(F(2)=6.42, p=0.003)$, mainly due to a difference between patients and controls (CTR vs SCZH : $p=0.002 ; C T R$ vs SCZH $\mathrm{SH}^{+} \mathrm{p}=0.03$; $\mathrm{SCZH}^{-}$vs SCZH $\left.{ }^{+}: \mathrm{p}=0.36\right)$. Hallucinators and non-hallucinators were also matched for duration of illness as well as severity of negative symptoms.

\section{Behavior}

Detection threshold estimates did not differ between groups (CTR: $-17.77 \pm 2.85 \mathrm{dBSNR}$; $\left.\mathrm{SCZH}^{-}:-19.29 \pm 4.69 \mathrm{dBSNR} ; \mathrm{SCZH}^{+}:-17.91 \pm 4.66 \mathrm{dBSNR} ; \mathrm{p}=0.90\right)$. There was also no difference between groups in the number of missed trials $(p=0.11)$.

In agreement with previous reports ${ }^{4,6}$, we found a significant effect of group on $\mathrm{CH}$ $\left(x^{2}(2)=7.68, \mathrm{p}=0.022\right)$, with $\mathrm{SCZH}^{+}$endorsing more $\mathrm{CH}$ than $\mathrm{SCZH}^{-}(\mathrm{z}=2.23, \mathrm{p}=0.026)$ and $\mathrm{CTR}$ $(z=2.53, p=0.011)$ (Figure 2A). No difference was observed between CTR and $\mathrm{SCZH}^{-}(p=0.86)$, or between CTR and SCZ (collapsed $\mathrm{SCZ}^{+}$and $\mathrm{SCZ}^{-}$groups) ( $\mathrm{p}=0.11$ ), indicating that the effect is specific to SCZs who hallucinate. Importantly, we also found a significant positive correlation between the percentage of endorsed $\mathrm{CH}$ and hallucination-severity in the whole sample $\left(r_{s}=0.341, p_{s}=0.008\right.$; remained significant after controlling for site $\left(r_{s, \text { site }}=0.326, p_{s, \text { site }}=0.013\right)$ and $\operatorname{diagnosis}\left(r_{s \text {,diagnosis }}=0.273, p_{s \text {, diagnosis }}=0.038\right)$ ) and in patients $\left(r_{s}=0.33, p_{s}=0.05\right)$ (Figure 2B).

Beyond no-tone trials, no significant differences were observed in the number of hits ('Yes' responses) at the 75\% ( $p=0.65)$ and $50 \%$ detection conditions $(p=0.42)$, while at the $25 \%$ 
$213 \mathrm{SCZH}^{+}$achieving more hits $(z=2.05, \mathrm{p}=0.041)$ (Figure S1). Additionally, we found no differences in 214 reported confidence between groups, but observed the predicted profile across conditions 215 (increase in reported confidence in 'Yes' responses (decrease in 'No' responses), from no-tone to $21675 \%$ detection condition) (Figure S2). Finally, we found a positive correlation between $\mathrm{CH}$ and 217 confidence ratings related to $\mathrm{CH}\left(r_{s}=0.310, p_{s}=0.018 ; r_{s, \text { site }}=0.285, p_{s, \text { site }}=0.032 ; r_{s}\right.$, diagnosis $=0.295$, ps,diagnosis $=0.026$ ): subjects who endorsed more $\mathrm{CH}$ also reported more confidence in their $\mathrm{CH}$

219 (Figure S3).

A.

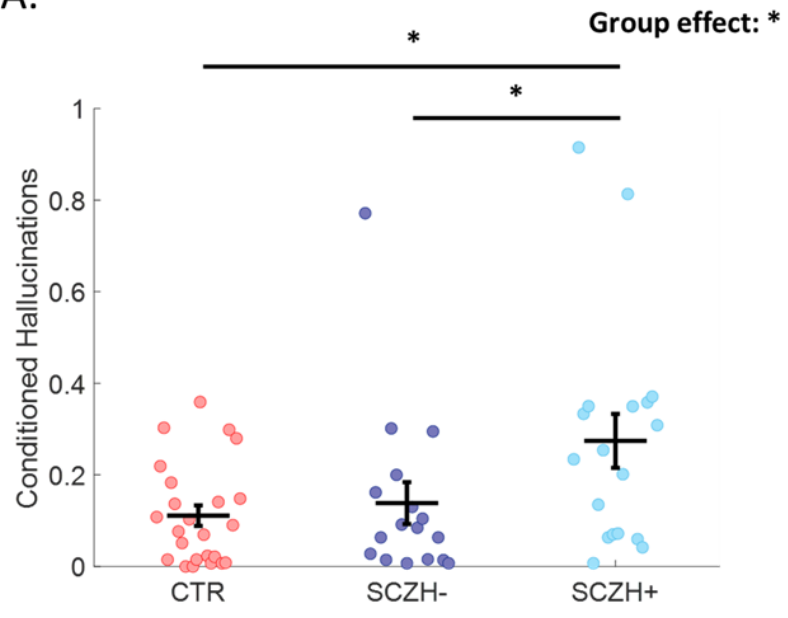

B.

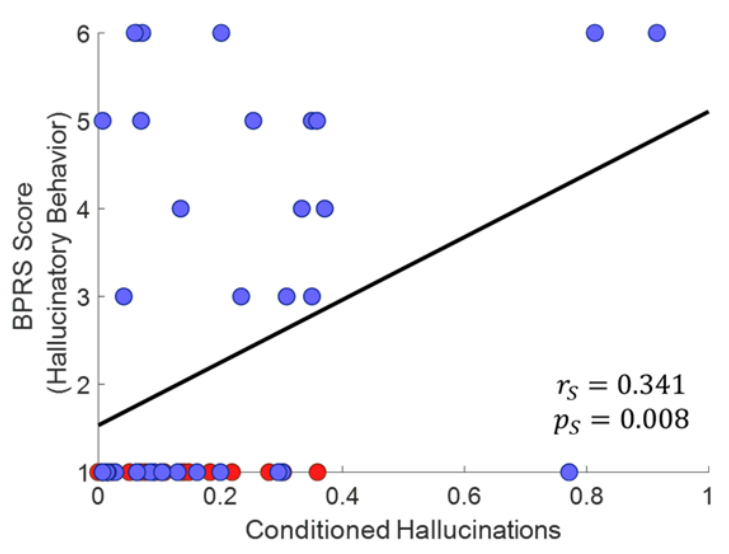

Figure 2: Conditioned hallucinations and clinical hallucinations. (A.) Conditioned hallucinations rate was

223 significantly elevated in $\mathrm{SCZH}^{+}$, compared to $\mathrm{SCZH}^{-}$or $\mathrm{CTR}$. Error bars represent $\pm 1 \mathrm{SEM}$. (B.) The 224 conditioned hallucinations rate correlated with clinical hallucination severity. Red: CTR; Blue: SCZ. 


\section{Computational modeling}

Model 4 (free parameters: $\omega_{2}, \beta$ and $v ; \omega_{3}$ was fixed; Figure 3A) emerged as a clear winner ( $\mathrm{PEP}_{4}=0.999$; groups did not differ in model fit) (Figure S4), consequently all the following results are with respect to this model, unless mentioned otherwise. Note that adding $v$ significantly increased log-model evidence, despite increasing the complexity of the model.

We found no significant differences in parameter estimates between groups $\left(\omega_{2}\right.$ : $\mathrm{p}=0,996 ; \beta: \mathrm{p}=0.20 ; v: \mathrm{p}=0.30)$ (Figure S5), except for a trend for lower $\beta$ in hallucinators $\left(\mathrm{SCZH}^{+}\right)$ as compared to non-hallucinators (CTR and $\mathrm{SCZH}^{-}$collapsed) $(\mathrm{p}=0.07)$. Nonetheless, we found significant correlations between $\mathrm{CH}$ and $\beta\left(r_{\mathrm{S}}=-0.72, \mathrm{p}_{\mathrm{s}}<0.001 ; \mathrm{r}_{\mathrm{s}, \text { site }}=-0.71, \mathrm{p}_{\mathrm{S}, \text { site }}<0.001\right.$; $\left.r_{s, \text { diagnosis }}=-0.72, p_{s, \text { diagnosis }}<0.001\right) \quad$ (Figure 3D) as well as $v \quad\left(r_{s}=0.59, \quad p_{s}<0.001 ; \quad r_{s, \text { site }}=0.56\right.$, $\mathrm{p}_{\mathrm{s}, \text { site }}<0.001 ; \mathrm{r}_{\mathrm{s} \text {, diagnosis }}=0.57, \mathrm{p}_{\mathrm{s} \text {, diagnosis }}<0.001$ ) (Figure 3B). In other words, endorsement of more $\mathrm{CH}$ was related with more stochastic decisions and stronger prior weighting (the 2 parameters were not correlated $\left.r_{s}=-0.10, p_{s}=0.45\right)$. Interestingly, although confidence ratings were not used to fit model parameters, we observed a correlation between confidence in reporting $\mathrm{CH}$ and $v$ $\left(r_{s}=0.46, p_{s}<0.001 ; r_{s, \text { site }}=0.44, p_{s, \text { site }}<0.001 ; r_{s, \text { diagnosis }}=0.44, p_{s, \text { diagnosis }}<0.001\right)$ (Figure 3C). Finally, with regard to belief trajectories, we found, that $\mathrm{SZH}^{+}$had a tendency to update their perceptual $\left(X_{1}\right)$ and contingency beliefs $\left(X_{2}\right)$ less $\left(X_{1}\right.$ : a trend for elevated $X_{1}$ beliefs in $\mathrm{SZH}^{+}$compared to CTR ( $p=0.097) ; X_{2}$ : a trend for elevated $X_{2}$ beliefs in $\mathrm{SZH}^{+}$compared to CTR $(p=0.069)$ and a significant block effect $(\mathrm{t}(409)=-2.15, \mathrm{p}=0.032) ; X_{3}$ : a significant block effect $(\mathrm{t}(409)=4.18$, $p<0.001)$ ). 
A.

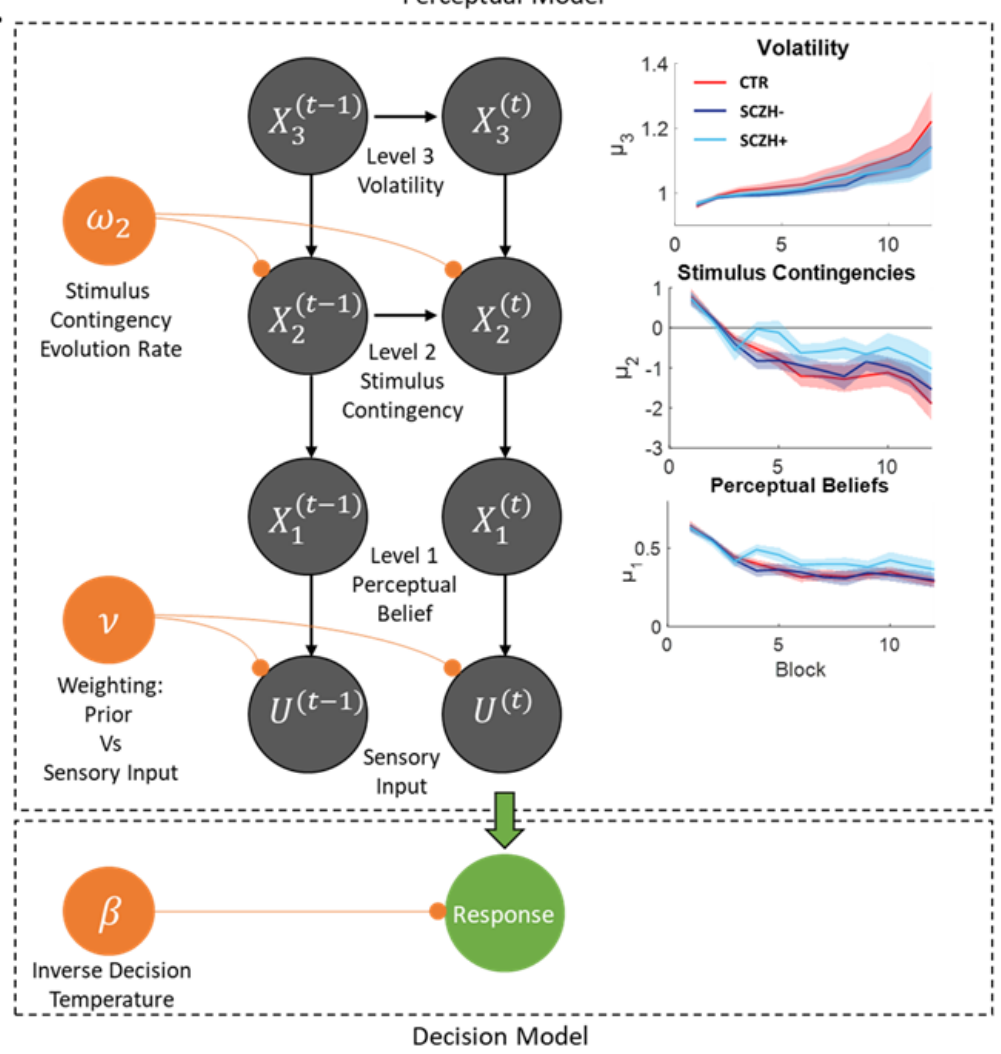

B.

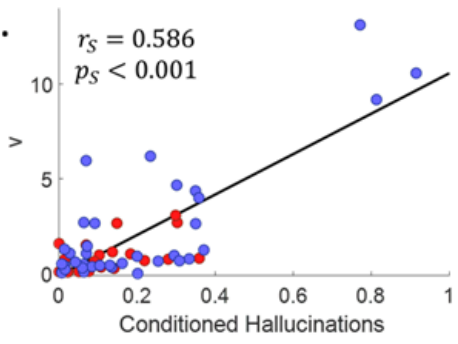

C.
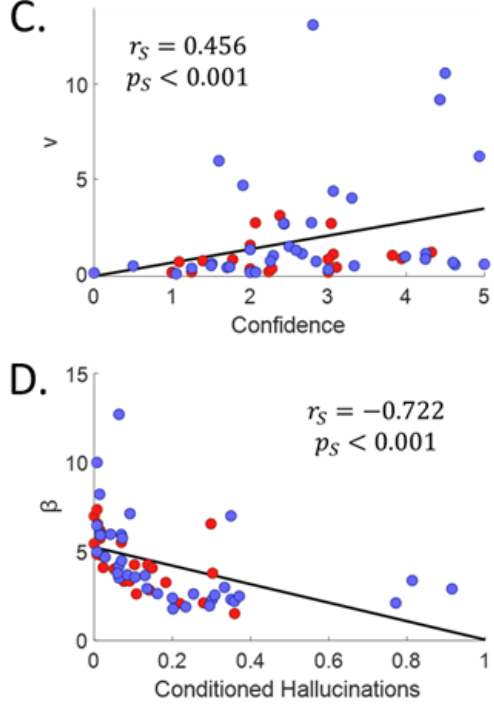

Figure 3: Hierarchical Gaussian Filter. (A.) Illustration of the (best-performing) HGF model (perceptual and decision component) that was fitted to behavioral data (left). Gray nodes correspond to states ( $U$ : Sensory inputs; $X_{1}$ : Probability of a tone being present; $X_{2}$ :Audiovisual association; $X_{3}$ : Volatility of the

251 audiovisual association), orange nodes to parameters $\left(\omega_{2} ; v ; \beta\right)$ and the green node to response/percept.

$252 \mathrm{SCZH}^{+}$showed a tendency towards reduced updating of their perceptual $\left(X_{1}\right)$ and contingency $\left(X_{2}\right)$ beliefs 253 (right - middle and lower panels). We found no group-effect on volatility estimates ( $X_{3}$; right upper panel). 254 Shaded areas represent \pm 1 SEM. (B,C,D.). Prior weighting $(v)$ correlated with $\mathrm{CH}$ rate $(\mathbf{B}$.) and $\mathrm{CH}$-related 255 confidence (C.). Inverse temperature ( $\beta$ ) correlated only with CH rate (D.). Red: CTR; Blue: SCZ 
Glutamate, strong priors and hallucinations

258

259

260

261

262

263

264

265

266

267

268

269

270

271

272

273

274

275

276

277

278

279

Glx levels were measured in four brain regions (LIN, ACC, LAC and rdIPFC) (Figure 4A-D).

We hypothesized that prior weights $(v)$ might be encoded in (or associated with) LIN $^{4}$. The following datasets were excluded from further analyses, due to poor spectral quality: 1 from the LAC (1 SCZH $)$, 2 from the ACC ( $\left.2 \mathrm{SCZH}^{+}\right)$and 2 from the rdIPFC ( $\left.2 \mathrm{SCZH}^{-}\right)$. Regional concentrations of Glx and spectral quality are presented in Table S2. We found no differences in Glx in any of the four regions (or in total GIx), except for a trend for lower Glx concentration in $\mathrm{SCZH}^{+}$in LIN (Figure S6). Furthermore, in agreement with our hypothesis, we observed a significant negative correlation between GIx concentration in LIN and the $v$ parameter $\left(r_{s}=-0.28, p_{s}=0.043 ; r_{s}\right.$,age $=-$ 0.30, $\mathrm{p}_{\mathrm{s}, \text { age }}=0.027$; but $\mathrm{r}_{\mathrm{s}, \mathrm{sit}}=-0.20, \mathrm{p}_{\mathrm{s}, \mathrm{site}}=0.14$ ), with stronger prior weighting (higher $v$ ) being associated with lower Glx levels (Figure 4E) (trend-wise correlation when controlling for diagnosis: $r_{\mathrm{s} \text {, diagnosis }}=-0.25, p_{\mathrm{s} \text {, diagnosis }}=0.068$ or medication: $r_{\mathrm{s}, \text { medication }}=-0.26, \mathrm{p}_{\mathrm{s} \text {, medication }}=0.056$; but significant when we also took into account age: $r_{s, \text { diagnosis,age }}=-0.28, p_{s, \text { diagnosis,age }}=0.046$;

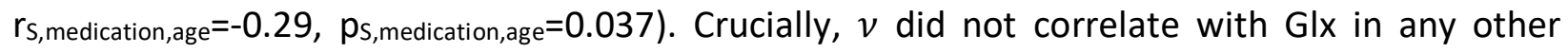
region (ACC: $r_{s}=-0.20, p_{s}=0.15$; LAC: $r_{s}=-0.16, p_{s}=0.24 ;$ rdIPFC: $r_{s}=0.16, p_{s}=0.24$; correlations remained non-significant after controlling for diagnosis and age) (Figure S7) or with total Glx ( $r_{S}=-$ $\left.0.12, \mathrm{p}_{\mathrm{s}}=0.39\right)$ and Glx in LIN did not correlate with $\beta\left(\mathrm{r}_{\mathrm{s}}=0.12, \mathrm{p}_{\mathrm{s}}=0.37\right), \mathrm{CH}\left(\mathrm{r}_{\mathrm{s}}=-0.21, \mathrm{p}_{\mathrm{s}}=0.12\right.$; trend-wise negative correlation when controlling for age: $\left.r_{s, a g e}=-0.24, p_{s, a g e}=0.081\right), B P R S-12\left(r_{s}=-\right.$ 0.19, $\left.p_{s}=0.18\right)$, age $\left(r_{s}=-0.17, p_{s}=0.22\right)$ or medication $\left(r_{s}=-0.14, p_{s}=0.32\right)$. Stepwise linear regressions with $v$ as dependent variable and the four Glx levels as potential predictors (with and without "site" as a predictor) or GIX in LIN as dependent variable and $\beta, v$ as potential predictors confirmed that the only significant predictor of $v$ is the Glx concentration in $\operatorname{LIN}(F(53)=4.2$, $\mathrm{p}=0.045)$. 

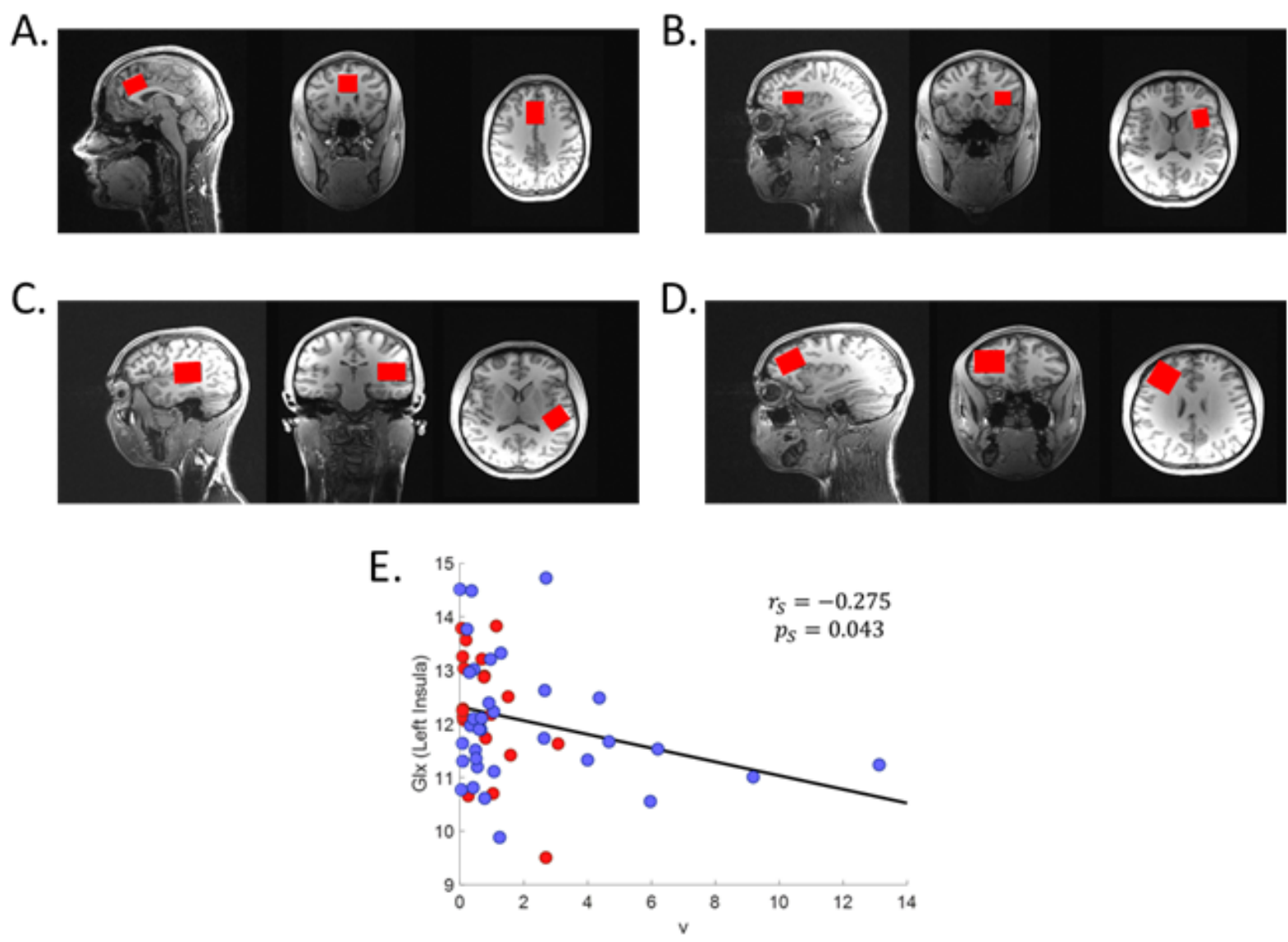

Figure 4: MR Spectroscopy - voxels and correlation with model parameters. (A-D.) Illustration of MRS and Glx level in LIN. Red: CTR; Blue: SCZ.

\section{Discussion}

Using a conditioned hallucinations task to probe prior-weighting ${ }^{4,6}$, and MRS to measure 288 glutamate levels we replicated and extended previous findings regarding the neurobiology of 
hallucinations. In a previous functional neuroimaging study insula cortex responses were associated with conditioned hallucinations and prior-weighting ${ }^{4}$. We found that patients who hallucinate endorse more $\mathrm{CH}$ in comparison to non-hallucinators and healthy controls, and $\mathrm{CH}$ rate correlated with ratings of hallucinations outside the laboratory, in agreement with previous reports $^{4,6}$. Additionally, participants who endorsed more $\mathrm{CH}$ exhibited stronger prior weighting (higher $v$ ) and more stochastic decisions (lower $\beta$ ), while hallucinators were more rigid with regard to their perceptual beliefs (flatter $X_{1}, X_{2}$ beliefs, i.e. reduced belief updating). Finally, we observed a negative correlation between prior-weighting and anterior insula Glx levels, but no relationship with sensory or association cortices.

Our findings are consistent with the results of previous functional imaging studies suggesting the engagement of anterior insula in $\mathrm{CH}^{4}$ and in $\mathrm{AVH}^{27,28}$. We speculate that the negative correlation between priors and insular Glx might be driven by a hypofunction of glutamatergic (NMDA) receptors situated on inhibitory interneurons, resulting in disinhibition of the anterior insula (also observed with ketamine infusion in healthy volunteers ${ }^{29}$ ). Future studies are needed to further explore the exact nature of the underlying biophysical mechanisms.

Subtle alterations in glutamate transmission and excitation / inhibition balance play a critical role in the pathophysiology of $\mathrm{SCZ}^{30-32}$. A mega-analysis suggested lower glutamate in $\mathrm{SCZ}$, perhaps as a function of medication status, with elevations in those who were more severely $\mathrm{ill}^{17}$. Some MRS studies have compared hallucinators with non-hallucinators, with mixed results. In sensory areas, a recent study found higher glutamate and glutamine in SCZ compared to $\mathrm{CTR}^{33}$ while another study found reduced Glu in the occipital cortex in SCZ and their healthy relatives, 
suggesting an association with illness liability ${ }^{34}$. Other studies found reduced Glx concentrations

311 in the auditory cortex in patients compared to healthy controls, but elevated Glx in the same 312 region in hallucinators compared to non-hallucinators ${ }^{18,19}$. Our study went a step further, 313 suggesting a functional interpretation to those neurochemical observations. We suggest that 314 glutamate levels in left anterior insula are associated with (and might encode) the precision of 315 prior distributions which, combined with sensory inputs, give rise to percepts. Low glutamate concentrations are related to overly-precise priors and hallucinations ${ }^{1}$.

Prior weighting correlated with glutamate levels specifically in LIN, and not in any other voxel (LAC, ACC or rdIPFC). Several studies have implicated insula in the pathophysiology and computational anatomy of hallucinations: Powers et al found that $\mathrm{CH}$ were associated with a stronger insula response in people who hear voices and had strong beliefs in the visual-auditory association during the task. Other studies have demonstrated a link between anterior insula and hallucinations, directly (e.g. ${ }^{27,35-38}$ ) or indirectly (e.g. ${ }^{39}$ ). That being said, other brain regions, not explored in the present study, might also be implicated in perceptual precision weighting. A good candidate is STS, for which a tripartite relation with $\mathrm{CH}$ and hallucinations has also been observed $^{4}$. Importantly, the lack of correlation between $v$ and global Glx can be interpreted as evidence against a global cortical glutamate theory of psychosis/hallucinations (in line with 327 predominant glutamatergic theories positing a widespread cortical NMDA hypofunction) and 328 more in favor of localized hypo-glutamatergia. 
331 appears incompatible with the present results. If high-level strong priors were driven by low-level 332 weak priors, we would expect significant but opposite associations between prior strength and 333 low-level (LAC) / high-level (LIN) glutamate concentrations. Instead, we only observed a negative 334 correlation between insular Glx and $v$, which is compatible with high-level prediction errors being 335 driven either by within-insula aberrant prediction errors, likely tethered to neuromodulators 336 (dopamine, acetylcholine or serotonin) ${ }^{2}$ or by abnormalities in a parallel hierarchy, e.g. weak egocentric corollary discharge signals ${ }^{40}$. For instance, low glutamate concentration might be the result of excessive striatal dopamine release, which has also been linked to overreliance on prior expectations ${ }^{41}$ and increased detection of missing tones in a conditioned hallucinations task ${ }^{5}$. current experiences and not on their history. A more careful examination of the psychotic symptoms' trajectory in a small subset of the participants (not presented here) revealed important variability even within subgroups, which might have obscured the most nuanced 344 differences. This might explain why we did not find group differences in model parameters or in 345 Glx levels (only trends), although medication and the moderate level of hallucinations (40\% of 346 hallucinators scored below 5 in BPRS-12) might have played a role too. Furthermore, three 347 participants exhibited very high $\mathrm{CH}$ rates ( $80 \%)$, a common behavior in $\mathrm{CH}$ tasks ${ }^{4,6}$. Despite 348 thorough training and exhaustive debriefing, it is difficult to know for certain whether those 349 outliers reflect extreme but meaningful cases of conditioned hallucinations or poor performance. 350 Finally, it is important to highlight the tentative nature of our MRS results: our hypothesis that 351 glutamate concentration in anterior insula (and not in any other region) might be related to prior 
352

353

354 355 attention.

356

357

358

359

360

361

362

363

364

365

366

367

368

369

370

371

372

373

374

375

376

9

0

7

72

weighting was entirely based on functional ( $\mathrm{fMRI}$ ) data ${ }^{4}$ and our result, although significant, would not survive a correction for multiple comparisons. Consequently, our conclusions, as well as the precise mechanisms underwriting the suggested associations, warrant further empirical

explanation of hallucinations, spanning behavior (conditioned hallucinations), information processing (strong priors) and neurochemistry (glutamate in LIN). Although this work bears further replication and extension, it provides neurochemical evidence to the relevance of prior beliefs to psychotic symptoms.

Conflict of interest: The authors report no conflicts of interest

Acknowledgements: This work was supported by R01MH12887 (JG/PRC), the Yale University Department of Psychiatry, the Connecticut Mental Health Center (CMHC) and Connecticut State Department of Mental Health and Addiction Services (DMHAS). The funders had no role in study design, data collection and analysis, decision to publish or preparation of the manuscript. We are grateful to Sarah Murphy for assistance with data collection. 


\section{References}

378 1. Corlett PR, Horga G, Fletcher PC, Alderson-Day B, Schmack K, Powers AR. Hallucinations and 379 Strong Priors. Trends Cogn Sci. 2019;23(2):114-127. doi:10.1016/j.tics.2018.12.001

380

381

382 383

384 385 386

2. Sterzer P, Adams RA, Fletcher P, et al. The Predictive Coding Account of Psychosis. Biol Psychiatry. 2018:1-10. doi:10.1016/j.biopsych.2018.05.015

3. Adams RA, Stephan KE, Brown HR, Frith CD, Friston KJ. The computational anatomy of psychosis. Front psychiatry. 2013;4(May):47. doi:10.3389/fpsyt.2013.00047

4. Powers AR, Mathys C, Corlett PR. Pavlovian conditioning-induced hallucinations result from overweighting of perceptual priors. Science (80- ). 2017;357(6351):596-600. doi:10.1126/science.aan3458

5. Schmack K, Bosc M, Ott T, Sturgill JF, Kepecs A. Striatal dopamine mediates hallucination-like perception in mice. Science (80- ). 2021;372(6537). doi:10.1126/science.abf4740

6. Kafadar E, Mittal VA, Strauss GP, et al. Modeling perception and behavior in individuals at clinical high risk for psychosis: Support for the predictive processing framework. Schizophr Res. 2020;226:167-175. doi:10.1016/j.schres.2020.04.017

7. Friston K. A theory of cortical responses. Philos Trans R Soc B Biol Sci. 2005;360(1456):815-836. doi:10.1098/rstb.2005.1622

8. Self MW, Kooijmans RN, Supèr H, Lamme VA, Roelfsema PR. Different glutamate receptors convey feedforward and recurrent processing in macaque V1. Proc Natl Acad Sci U S A. 2012;109(27):11031-11036. doi:10.1073/pnas.1119527109

9. Krystal JH, Karper LP, Seibyl JP, et al. Subanesthetic effects of the noncompetitive NMDA antagonist, ketamine, in humans: Psychotomimetic, perceptual, cognitive and neuroendocrine responses. Arch Gen Psychiatry. 1994;51:199-214.

10. Moghaddam B, Adams B, Verma A, Daly D. Activation of glutamatergic neurotransmission by ketamine: A novel step in the pathway from NMDA receptor blockade to dopaminergic and cognitive disruptions associated with the prefrontal cortex. J Neurosci. 1997;17(8):2921-2927. doi:10.1523/jneurosci.17-08-02921.1997 
Glutamate, strong priors and hallucinations

404

405

406

407

408

409

410

411

412

413

414

415

416

417

418

419

420

421

422

423

424

425

426

427

428

429

430

431

11. Corlett PR, Honey GD, Krystal JH, Fletcher PC. Glutamatergic model psychoses: Prediction error, learning, and inference. Neuropsychopharmacology. 2011;36(1):294-315. doi:10.1038/npp.2010.163

12. Corlett PR, Honey GD, Fletcher PC. Prediction error, ketamine and psychosis: An updated model. J Psychopharmacol. 2016;30(11):1145-1155. doi:10.1177/0269881116650087

13. Corlett PR, Honey GD, Fletcher PC. From prediction error to psychosis: Ketamine as a pharmacological model of delusions. J Psychopharmacol. 2007;21(3):238-252. doi:10.1177/0269881107077716

14. Sydnor VJ, Roalf DR. A meta-analysis of ultra-high field glutamate, glutamine, GABA and glutathione 1HMRS in psychosis: Implications for studies of psychosis risk. Schizophr Res. 2020;226:61-69. doi:10.1016/j.schres.2020.06.028

15. Marsman A, Van Den Heuvel MP, Klomp DWJ, Kahn RS, Luijten PR, Hulshoff Pol HE. Glutamate in schizophrenia: A focused review and meta-analysis of 1H-MRS studies. Schizophr Bull. 2013;39(1):120-129. doi:10.1093/schbul/sbr069

16. Wijtenburg SA, Wright SN, Korenic SA, et al. Altered Glutamate and Regional Cerebral Blood Flow Levels in Schizophrenia: A 1 H-MRS and pCASL study. Neuropsychopharmacology. 2017;42(2):562-571. doi:10.1038/npp.2016.172

17. Merritt K, McGuire PK, Egerton A, et al. Association of Age, Antipsychotic Medication, and Symptom Severity in Schizophrenia with Proton Magnetic Resonance Spectroscopy Brain Glutamate Level: A Mega-analysis of Individual Participant-Level Data. JAMA Psychiatry. 2021;78(6):667-681. doi:10.1001/jamapsychiatry.2021.0380

18. Hugdahl K, Craven AR, Nygård $\mathrm{M}$, et al. Glutamate as a mediating transmitter for auditory hallucinations in schizophrenia: A 1H MRS study. Schizophr Res. 2015;161(2-3):252-260. doi:10.1016/j.schres.2014.11.015

19. Hjelmervik H, Craven AR, Sinceviciute I, et al. Intra-Regional Glu-GABA vs Inter-Regional Glu-Glu Imbalance: A 1H-MRS Study of the Neurochemistry of Auditory Verbal Hallucinations in Schizophrenia. Schizophr Bull. 2020;46(3):633-642. doi:10.1093/schbul/sbz099

20. Corlett PR, Fletcher PC. Computational psychiatry: A Rosetta Stone linking the brain to mental 
Glutamate, strong priors and hallucinations

illness. The Lancet Psychiatry. 2014;1(5):399-402. doi:10.1016/S2215-0366(14)70298-6

21. Corlett PR, Schoenbaum G. Leveraging Basic Science for the Clinic-From Bench to Bedside. JAMA Psychiatry. 2020:1-4. doi:10.1001/jamapsychiatry.2020.3656

22. Hugdahl K, Sommer IE. Auditory verbal hallucinations in schizophrenia from a levels of explanation perspective. Schizophr Bull. 2018;44(2):234-241. doi:10.1093/schbul/sbx142

23. Mathys C, Daunizeau J, Friston KJ, Stephan KE. A Bayesian foundation for individual learning under uncertainty. Front Hum Neurosci. 2011;5(May):1-20. doi:10.3389/fnhum.2011.00039

24. Mathys CD, Lomakina El, Daunizeau J, et al. Uncertainty in perception and the Hierarchical Gaussian Filter. Front Hum Neurosci. 2014;8(November):1-24. doi:10.3389/fnhum.2014.00825

25. Kalman RE. A New Approach to Linear Filtering and Prediction Problems. J basic Eng. 1960;82(Series D):35-45.

26. Rigoux L, Stephan KE, Friston KJ, Daunizeau J. Bayesian model selection for group studies Revisited. Neuroimage. 2014;84:971-985. doi:10.1016/j.neuroimage.2013.08.065

27. Jardri R, Pouchet A, Pins D, Thomas P. Cortical Activations During Auditory Verbal Hallucinations in Schizophrenia: A Coordinate-Based Meta-Analysis. Am J Psychiatry. 2011;168:73-81.

28. Zmigrod L, Garrison JR, Carr J, Simons JS. The neural mechanisms of hallucinations: A quantitative meta-analysis of neuroimaging studies. Neurosci Biobehav Rev. 2016;69:113-123. doi:10.1016/j.neubiorev.2016.05.037

29. Anticevic A, Gancsos M, Murray JD, et al. NMDA receptor function in large-scale anticorrelated neural systems with implications for cognition and schizophrenia. Proc Natl Acad Sci. 2012;109(41):16720-16725. doi:10.1073/pnas.1208494109//DCSupplemental.www.pnas.org/cgi/doi/10.1073/pnas.1208494109

30. Jardri R, Hugdahl K, Hughes M, et al. Are Hallucinations Due to an Imbalance Between Excitatory and Inhibitory Influences on the Brain? Schizophr Bull. 2016;42(5):1124-1134. doi:10.1093/schbul/sbw075

31. Corlett PR. I Predict, Therefore I Am: Perturbed Predictive Coding Under Ketamine and in Schizophrenia. Biol Psychiatry. 2017;81(6):465-466. doi:10.1016/j.biopsych.2016.12.007 
Glutamate, strong priors and hallucinations

459

460

461

462

463

464

465

466

467

468

469

470

471

472

473

474

475

476

477

478

479

480

481

482

483

484

485

32. Howes O, McCutcheon R, Stone J. Glutamate and dopamine in schizophrenia: An update for the 21st century. J Psychopharmacol. January 2015. doi:10.1177/0269881114563634

33. Wijtenburg SA, West J, Korenic SA, et al. Multimodal Neuroimaging Study of Visual Plasticity in Schizophrenia. Front Psychiatry. 2021;12(April):1-8. doi:10.3389/fpsyt.2021.644271

34. Thakkar KN, Rösler L, Wijnen JP, et al. 7T Proton Magnetic Resonance Spectroscopy of GammaAminobutyric Acid, Glutamate, and Glutamine Reveals Altered Concentrations in Patients With Schizophrenia and Healthy Siblings. Biol Psychiatry. 2017;81(6):525-535.

doi:10.1016/j.biopsych.2016.04.007

35. Sommer IEC, Diederen KMJ, Blom JD, et al. Auditory verbal hallucinations predominantly activate the right inferior frontal area. Brain. 2008;131(12):3169-3177. doi:10.1093/brain/awn251

36. Hoffman RE. Revisiting arieti's "listening attitude" and hallucinated voices. Schizophr Bull. 2010;36(3):440-442. doi:10.1093/schbul/sbq025

37. Nanda S, Priya K, Khan T, et al. Combined Parietal-Insular-Striatal Cortex Stroke with New-Onset Hallucinations: Supporting the Salience Network Model of Schizophrenia. Psychiatry J. 2020:1-6. doi:10.1155/2020/4262050

38. Wylie K, Tregellas J. The role of the insula in schizophrenia. Schizophr Res. 2010;123(2-3):93-104. doi:10.1007/978-3-319-75468-0_27

39. Iannotti GR, Orepic P, Brunet D, et al. EEG Spatiotemporal Patterns Underlying Self-other Voice Discrimination. Cereb Cortex. 2021:1-15. doi:10.1093/cercor/bhab329

40. Leptourgos P, Corlett PR. Embodied Predictions, Agency, and Psychosis. Front Big Data. 2020;3(27):1-13. doi:10.3389/fdata.2020.00027

41. Cassidy CM, Balsam PD, Weinstein JJ, et al. A Perceptual Inference Mechanism for Hallucinations Linked to Striatal Dopamine. Curr Biol. 2018:1-12. doi:10.1016/j.cub.2017.12.059

42. Watson AB, Pelli DG. QUEST: A bayesian adaptive psychometric method. Percept Psychophys. 1983;33(2):113-120. doi:10.1167/17.3.10

43. Treutwein B, Strasburger H. Fitting the psychometric function. Percept Psychophys. 1999;61(1):87-106. doi:10.3758/BF03211951 
Glutamate, strong priors and hallucinations

486

487

488

489

490

491

492

493

494

495

496

497

498

499

500

501

502

503

504

505

506

507
44. Provencher SW. Estimation of metabolite concentrations from localized in vivo proton NMR spectra. Magn Reson Med. 1993;30(6):672-679. doi:10.1002/mrm.1910300604

45. Provencher SW. Automatic quantitation of localized in vivo $1 \mathrm{H}$ spectra with LCModel. NMR Biomed. 2001;14(4):260-264. doi:10.1002/nbm.698

46. Soher BJ, Young K, Bernstein A, Aygula Z, Maudsley AA. GAVA: Spectral simulation for in vivo MRS applications. J Magn Reson. 2007;185(2):291-299. doi:10.1016/j.jmr.2007.01.005

47. Gasparovic C, Neeb H, Feis DL, et al. Quantitative spectroscopic imaging with in situ measurements of tissue water T1, T2, and density. Magn Reson Med. 2009;62(3):583-590. doi:10.1002/mrm.22060

48. Korenic SA, Klingaman EA, Wickwire EM, et al. Sleep quality is related to brain glutamate and symptom severity in schizophrenia. J Psychiatr Res. 2020;120(April 2019):14-20.

doi:10.1016/j.jpsychires.2019.10.006 


\section{Supplementary Methods}

509 CH task

Prior to the experiment, participants completed a short practice session which was

511 repeated until their responses were at $85 \%$ detection accuracy. The main experiment took place

512 in an MRI scanner and consisted of two parts. First, we determined individual $75 \%$ detection

513 thresholds, using the QUEST maximum likelihood-based procedure for threshold estimation ${ }^{42}$.

514 This was implemented in two 40-trial initially-ascending interleaved staircases, with step-size

515 determined by QUEST based upon participant responses. We also estimated $25 \%$ and $50 \%$

516 detection-likelihood tone intensities, by fitting a psychometric function to the QUEST-computed

$51775 \%$ likelihood of detection of noise-embedded tone (Figure 1B, left) ${ }^{43}$.

518 Throughout the experiment, participants were instructed to keep their eyes on a fixation 519 cross. Trials were structured as follows (Figure 1A): there was a 500- to 1000-ms fixation from 520 trial start, followed by the simultaneous presentation of the checkerboard and the tone (if 521 present), for 1 s. Participant responses were recorded for 1000 to 1500 ms after stimulus offset. 522 During the twelve main blocks, there was an additional 2000-ms period to record confidence523 rating response, during which participants could hold down the response button to indicate their 524 confidence level. 
A white visual fixation cross was present on a black background throughout the experiment. The visual stimulus was a $4 \times 7$ gray-on black checkerboard pattern, with gray squares at $25 \%$ brightness to maximize visual stimulation and minimize after-effect. The auditory stimulus consisted of a 1-kHz pure tone with a 100-ms tapered envelope to prevent transient effects and was presented via magnetic-resonance-compatible supra-aural headphones (Resonance Technology Corporation, Model\#650-060). Participants were provided with foam earplugs for additional protection from scanner noise, which was estimated to be at 130dB SPL. In order to provide a consistent acoustic background for our auditory detection experiment, broadband white noise was presented at $130 \mathrm{~dB}$ SPL; given the 40-dB attenuation afforded by the headphones, this was estimated to correspond to a level that was 40dB SPL louder than the scanner noise.

\section{H-MR Spectroscopy}

We used Siemens 3T PRISMA MRI systems equipped with a 32-channel phase array head coils. Spectra were acquired from voxels encompassing the anterior cingulate cortex $(2 \times 2 \times 1.5$ $\mathrm{cm})(\mathrm{ACC})$, left anterior insula $(2 \times 1.5 \times 1.5 \mathrm{~cm})(\mathrm{LIN})$, right dorsolateral PFC $(2 \times 2 \times 2 \mathrm{~cm})(\mathrm{rdIPFC})$, and left auditory cortex $(2.5 \times 1.5 \times 2.0 \mathrm{~cm})($ LAC) (see Figure 4A-D) using PRESS localization $(T R / T E=2000 / 30 m s, N E X=128$ for the anterior cingulate, and 256 for other voxels, $2500-\mathrm{Hz}$ spectral width, 2048 complex points). Water unsuppressed spectra (NEX=16) were acquired for referencing, as well for phase and eddy current correction. Spectra were analyzed with LCModel $^{44,45}$ using a basis set that included alanine, aspartate, creatine, $\boldsymbol{\gamma}$-aminobutyric acid, 
548 glucose, glutamate (Glu), glutamine (Gln), glutathione, glycine, glycerophosphocholine, lactate, 549 myo-Inositol, N-acetylaspartate, N-acetylaspartylglutamate, phosphocholine, phosphocreatine, 550 phosphoroylethanolamine, scyllo-Inositol, and taurine generated in GAVA ${ }^{46}$. Spectra were 551 visually inspected for quality and only spectra with SNR $>10$ and water FWHM $<0.1$ ppm were 552 included. Metabolites were referenced to water and only metabolites with good fits (i.e. percent 553 standard deviation, Cramer-Rao lower bounds < 20\%) were included. Metabolites were 554 referenced to water and presented in institutional units (I.U.) with values corrected for the 555 proportion of the gray matter, white matter, and cerebrospinal fluid (CSF) water content within 556 each spectroscopic voxel, and metabolite and water relaxation times according to ${ }^{47}$ using in557 house Matlab code utilized in other studies 33,48

\section{Statistical analysis - Behavior}

We compared the behavior of the different groups using exclusively non-parametric statistics. Between-group differences $\left(\mathrm{CTR}\right.$ vs $\mathrm{SCZH}^{-}$vs $\left.\mathrm{SCZH}^{+}\right)$were assessed using the Kruskal

Wallis $\mathrm{H}$ test. When necessary, we performed post-hoc 2-by-2 comparisons using the Mann-

563 Whitney U test. All the analyses were two-tailed. Spearman's correlation coefficients were used 564 to assess the relationships between the main behavioral variables ( $\mathrm{CH}$ and confidence) and with 565 hallucination-severity (BPRS-12 score). Given that we adopt a dimensional approach to 566 hallucinations (psychosis) and in agreement with previous studies ${ }^{4,6}$, we pooled data across 567 groups. Spearman's partial correlations were used to control for confounders (site (CMHC vs 568 MPRC) and diagnosis (CTR vs SCZ)). 


\section{Statistical analysis - Computational modeling}

The Kruskal Wallis $\mathrm{H}$ test and the Mann-Whitney $\mathrm{U}$ test were used to compare model-

parameters across groups. Spearman's correlation coefficients were used to assess the

relationships between model parameters and behavioral data / hallucination severity (and partial compared using linear mixed-effects models comprising group and block (linear part of the trajectories, i.e. blocks 6-12; see also Figure 1B, right) as fixed effects together with Gaussian

577 random effects for intercepts and slopes.

\section{Statistical analysis - MRS} groups. We hypothesized that strong priors might be underscored by altered levels of Glx in LIN.

To test this hypothesis, we computed Spearman's correlation coefficients between Glx in LIN and the $v$ HGF parameter. As a control (exploratory analysis), we also computed the correlations between GIx in other regions (ACC, LAC and rdIPFC; we also looked at total GIx) and $v$, as well as

585 between Glx in LIN and $\beta, \mathrm{CH}$, hallucination severity, age and medication (CPZ equivalents).

586 Partial correlations were also used to control for confounders. To further test the specificity of 587 the dependence of strong priors on LIN, we performed two stepwise regressions with 588 bidirectional elimination and computed the $p$-values for the F-statistics of the change in the 
Glutamate, strong priors and hallucinations

589

590

591 intercept) or a full (intercept + Glx in all four regions) model. To control for the effect of site, we

592 $593 \quad$ variable and $\beta, v$ as potential predictors.

residual sum of squares that results from adding or removing a term: 1 . With $v$ as the dependent variable and Glx in the four regions as potential predictors, starting from a constant (only repeated the stepwise regression adding site as a predictor. 2. With GIX (LIN) as the dependent
594

595

596

597

598

599

600

601

602

603

604

605

.

.

(

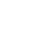




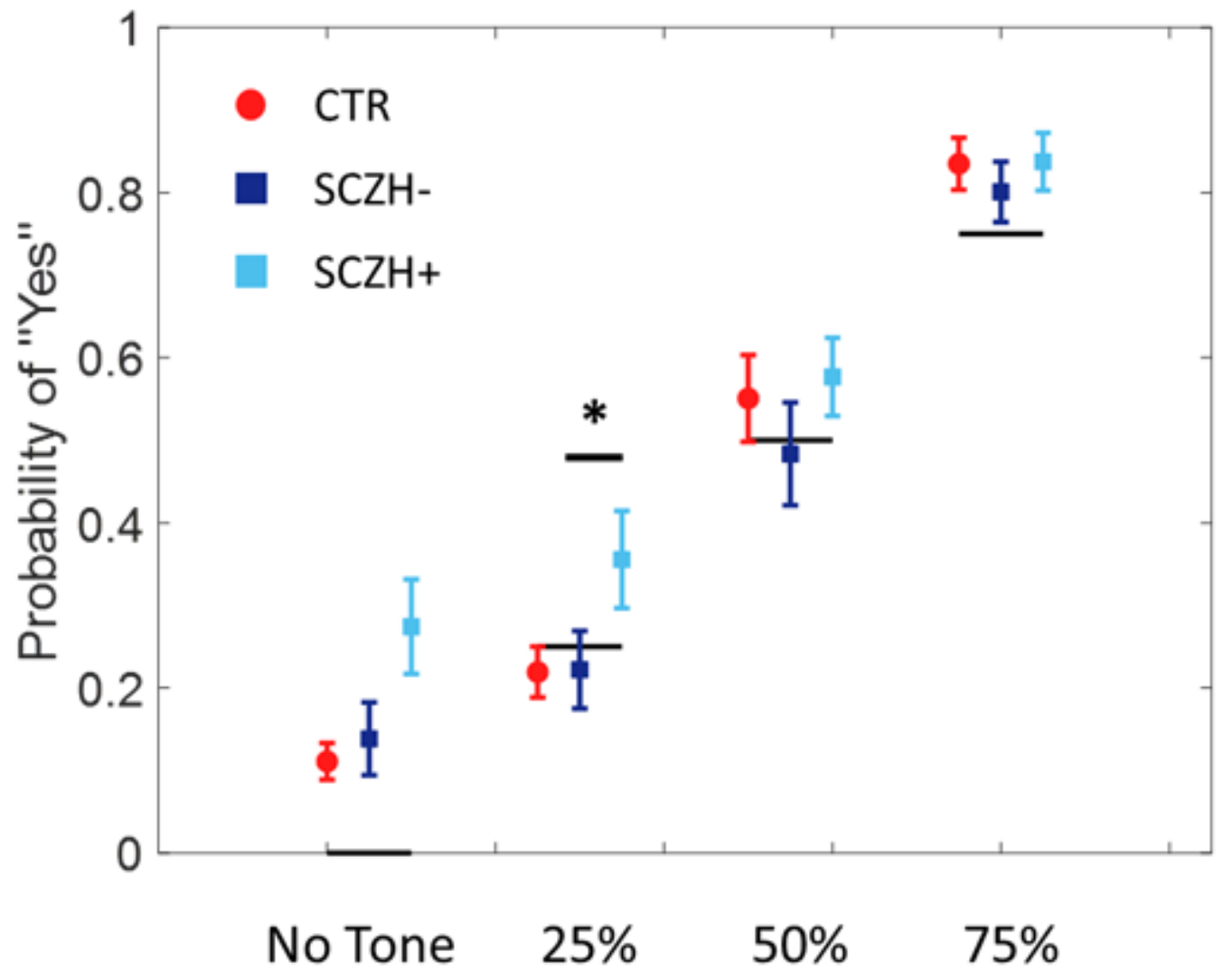

607

608 Figure S1: Hits and false alarms. Group differences were observed only in the No-tone (see Figure 1) and $60925 \%$ detection-rate conditions $\left(\mathrm{SCZH}^{+}\right.$vs SCZH $)$. Error bars represent \pm 1 SEM. 
Glutamate, strong priors and hallucinations

611

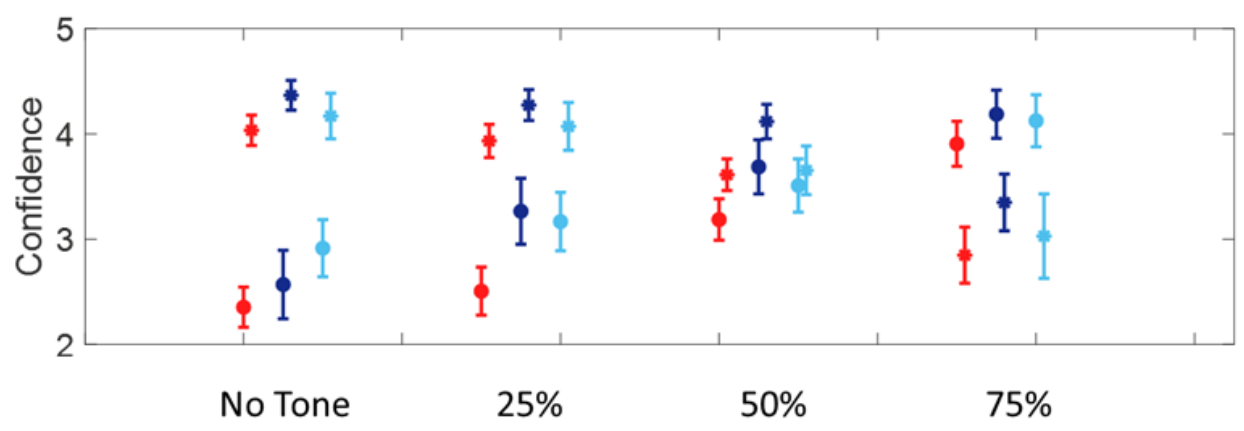

- Yes - CTR

* No - CTR

- Yes-SCZH-

* No-SCZH-

- $\mathrm{Yes}-\mathrm{SCZH}+$

* $\mathrm{No}-\mathrm{SCZH}+$

612 Figure S2: Confidence across conditions. We observed no significant group-differences for either

613 response (positive or negative). Confidence when responding positively increased from left (No-tone) to

614 right (75\%). The opposite was true for negative responses. Error bars represent \pm 1 SEM.

615 


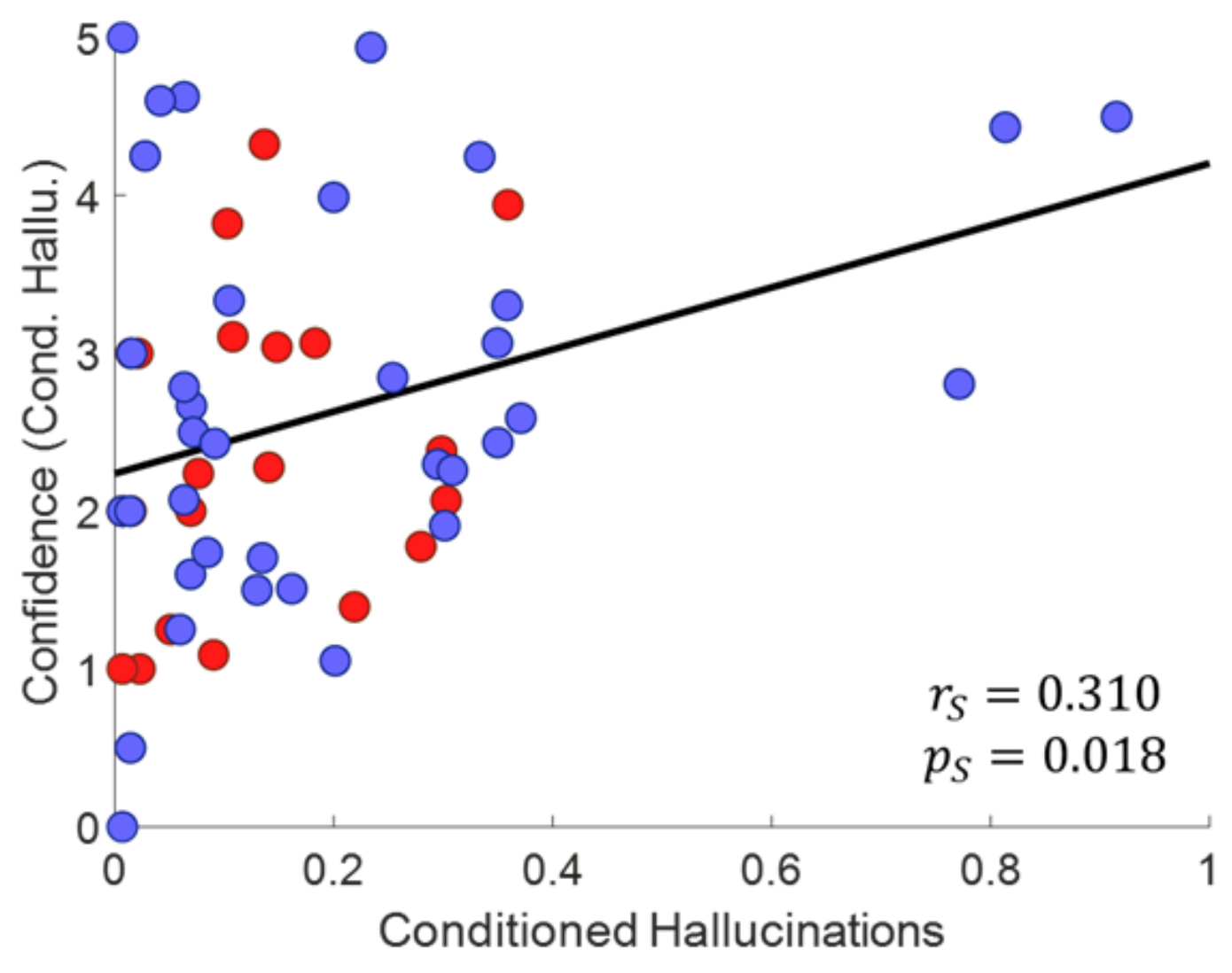

617 Figure S3: Correlation between conditioned hallucinations and confidence in reporting conditioned 618 hallucinations. Red: CTR; Blue: SCZ. 
Glutamate, strong priors and hallucinations

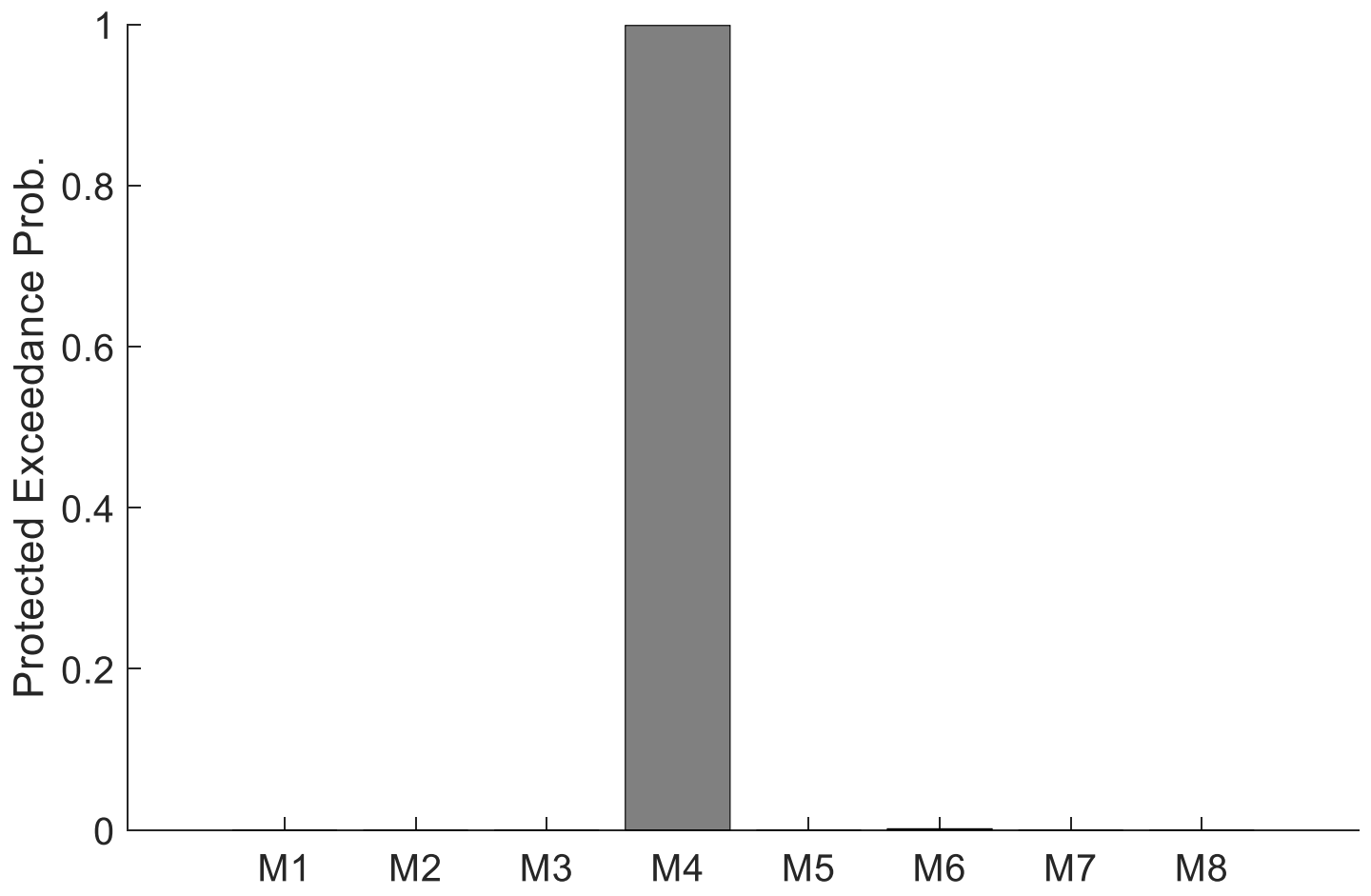

620

621 Figure S4: Bayesian model selection based on protected exceedance probability. Model 4 (3 free

622 parameters: $\omega_{2}, v, \beta$ ) clearly outperformed the other models (see Table S3 for more details).

623

624

625

626 
Glutamate, strong priors and hallucinations

627
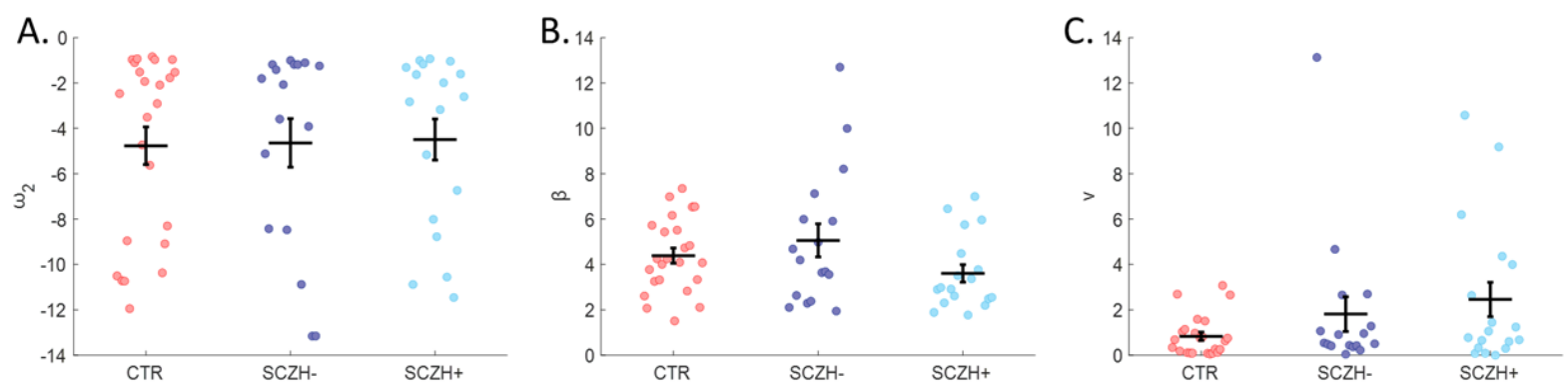

628 Figure S5: Model parameters. Estimated parameters didn't differ across groups. Error bars represent \pm 1

629 SEM.

630

631 
Glutamate, strong priors and hallucinations

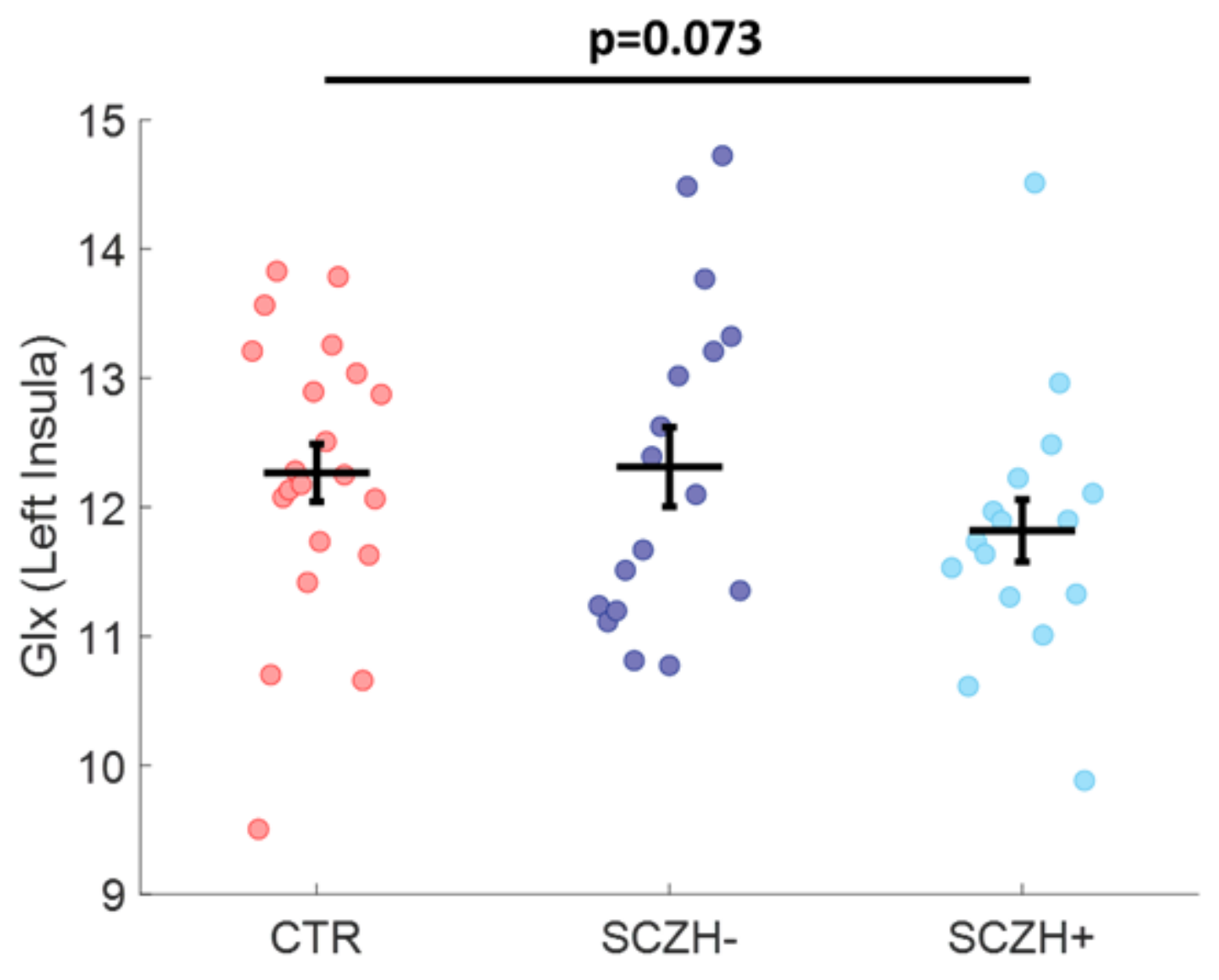

632

633 Figure S6: Glx concentration in LIN. We observed a trend towards reduced Glx in SCZH ${ }^{+}$Error bars 634 represent \pm 1 SEM.

635

636 
Glutamate, strong priors and hallucinations
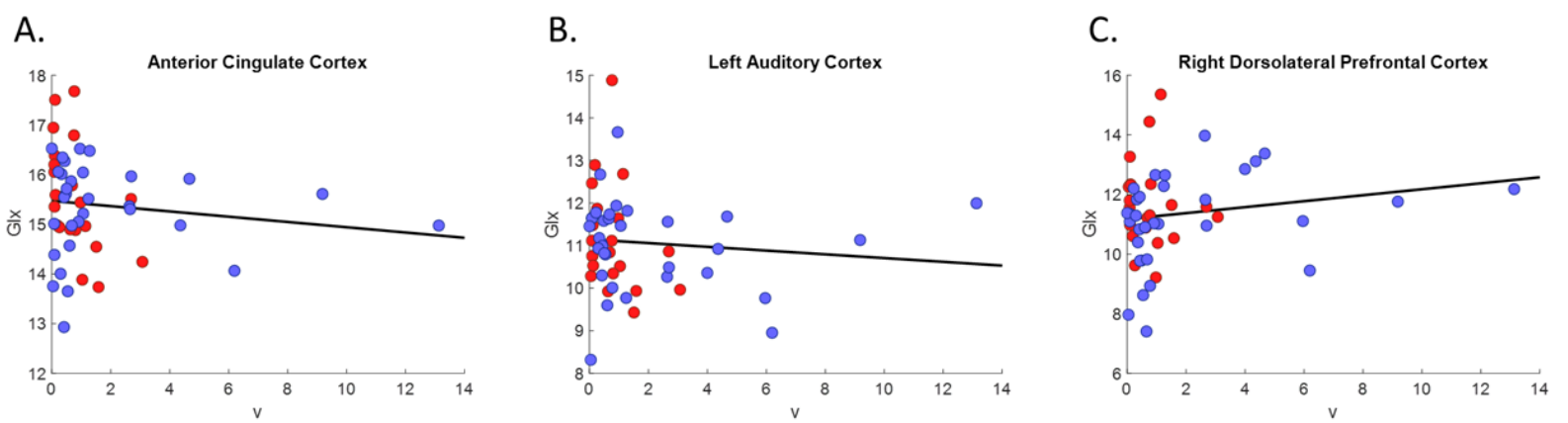

637

638 Figure S7: Correlation between prior weighting $(v)$ and the Glx concentration in ACC, LAC and rdIPFC.

639 None of the correlations was significant. Red: CTR; Blue: SCZ.

640

641

642

643

644

645

646

647

648

649

650

651 
Glutamate, strong priors and hallucinations

Table S1: MRS Participant Characteristics (mean (SD))

\begin{tabular}{|c|c|c|c|c|c|}
\hline Variables & CTR $(n=21)$ & $\mathrm{SCZH}^{-}(\mathrm{n}=17)$ & $\mathrm{SCZH}^{+}(\mathrm{n}=16)$ & Statistic & p value \\
\hline Age & $35.9(10.6)$ & $31.3(9.6)$ & $34.8(10.1)$ & $F=1.02$ & 0.37 \\
\hline Gender & $9 / 12$ & $11 / 6$ & $11 / 5$ & $\chi^{2}=3.03$ & 0.22 \\
\hline Race (African American/Caucasian/other/missing) & $3|15| 2 \mid 1$ & $8|9| 0 \mid 0$ & $3|11| 1 \mid 1$ & $\chi^{2}=7.42$ & 0.28 \\
\hline Participant Education & $15.7(2.0)$ & $14.1(1.2)$ & $14.5(1.7)$ & $F=4.78$ & 0.01 \\
\hline Maternal Education & $14.6(4.2)$ & $14.5(2.4)$ & $14.4(4.5)$ & $F=0.01$ & 0.99 \\
\hline Paternal Education & $14.5(4.6)$ & $14.0(4.7)$ & $14.0(6.9)$ & $F=0.10$ & 0.90 \\
\hline \multicolumn{6}{|l|}{ Neurocognitive test results } \\
\hline WTAR & $108.9(28.7)$ & $101.2(28.5)$ & $112.7(9.2)$ & $F=0.92$ & 0.41 \\
\hline MD Processing Speed & $51.0(17.9)$ & $42.5(14.4)$ & $47.8(11.9)$ & $F=1.43$ & 0.25 \\
\hline MD Working Memory & $52.6(13.9)$ & $43.1(15.2)$ & $49.0(12.3)$ & $F=2.17$ & 0.12 \\
\hline MD Verbal Learning & $46.1(12.1)$ & $42.9(14.5)$ & $44.7(12.0)$ & $F=0.27$ & 0.76 \\
\hline \multicolumn{6}{|l|}{ Clinical Ratings } \\
\hline BPRS Hallucinatory Behavior & $1.0(0.0)$ & $1.0(0.0)$ & $4.6(1.2)$ & - & - \\
\hline BPRS Delusions & $2.0(0.0)$ & $3.6(1.8)$ & $5.8(1.8)$ & $t=3.40^{1}$ & $0.002^{1}$ \\
\hline BPRS Negative Symptoms & $3.4(1.2)$ & $6.1(2.4)$ & $4.9(1.7)$ & $t=-1.68^{1}$ & $0.09^{1}$ \\
\hline Duration of Illness (yrs) & & $8.4(8.3)$ & $12.7(11.2)$ & $t=1.23$ & 0.23 \\
\hline \multicolumn{6}{|l|}{ Medication } \\
\hline Antipsychotic (Atypical |Typical) & & $16 \mid 1$ & $14 \mid 2$ & $\chi^{2}=0.44$ & 0.51 \\
\hline Antipsychotic medication: CPZ & alents (mg) & $278.4(259.5)$ & $249.9(198.6)$ & $t=-0.35$ & 0.73 \\
\hline \multicolumn{6}{|c|}{$\begin{array}{l}\text { Antidepressant }+ \text { Benzodiazepine }+ \text { Mood } \\
\text { stabilizer }\end{array}$} \\
\hline Antidepressant+ Benzodiazepir & & 0 & 3 & & \\
\hline Benzodiazepine & & 0 & 3 & & \\
\hline Antidepressant & & 4 & 3 & & \\
\hline Mood stabilizer & & 3 & 0 & & \\
\hline
\end{tabular}

WTAR=Wechsler Test of Adult Reading, measure of premorbid intelligence; MD Working Memory= Working memory domain (MATRICS); MD Processing Speed= processing speed domain (MATRICS); MD Verbal Learning=verbal learning domain (MATRICS); $\quad \mathrm{CPZ}=$ chlorpromazine equivalent; $\mathrm{BPRS}$ Delusions=Suspiciousness+Unusual Thought Content; BPRS Negative Symptoms=Emotional Withdrawal+Motor Retardation+Blunted Affect;

${ }^{1} \mathrm{SCZH}^{-}$vs. SCZH 


\begin{tabular}{|c|c|c|c|c|}
\hline Variables & CTR $(n=21)$ & $\mathrm{SCZH}^{-}(\mathrm{n}=17)$ & $\mathrm{SCZH}^{+}(\mathrm{n}=16)$ & $p$ value ${ }^{1}$ \\
\hline \multicolumn{5}{|l|}{ LIN } \\
\hline Glx & 12.27 (1.09) & $12.31(1.26)$ & $11.82(1.03)$ & 0.27 \\
\hline Glx fits (\%SD) & $4.62(0.67)$ & $4.71(1.05)$ & $4.38(0.62)$ & 0.49 \\
\hline FWHM & $\begin{array}{c}0.0337 \\
(0.0065)\end{array}$ & $\begin{array}{c}0.0343 \\
(0.0099)\end{array}$ & $\begin{array}{c}0.0335 \\
(0.0078)\end{array}$ & 0.96 \\
\hline SNR & $35.76(6.90)$ & $36.71(8.54)$ & 37.69 (7.03) & 0.68 \\
\hline \multicolumn{5}{|l|}{$A C C$} \\
\hline Glx & 15.54 (1.10) & $15.37(1.04)$ & $15.21(0.77)$ & 0.71 \\
\hline Glx fits (\%SD) & $3.76(0.54)$ & $3.94(0.24)$ & $3.93(0.27)$ & 0.26 \\
\hline FWHM & $\begin{array}{c}0.0257 \\
(0.0035)\end{array}$ & $\begin{array}{c}0.0282 \\
(0.0083)\end{array}$ & $\begin{array}{c}0.0277 \\
(0.0048)\end{array}$ & 0.46 \\
\hline SNR & $42.43(6.85)$ & 43.71 (7.09) & $42.57(4.13)$ & 0.64 \\
\hline \multicolumn{5}{|l|}{$L A C$} \\
\hline Glx & $11.16(1.26)$ & $11.34(1.16)$ & $10.79(0.86)$ & 0.25 \\
\hline Glx fits (\%SD) & $4.90(1.09)$ & $4.94(0.85)$ & $4.50(0.89)$ & 0.28 \\
\hline FWHM & $\begin{array}{c}0.0382 \\
(0.0077)\end{array}$ & $\begin{array}{c}0.0407 \\
(0.0136)\end{array}$ & $\begin{array}{c}0.0374 \\
(0.0103)\end{array}$ & 0.63 \\
\hline SNR & 47.95 (14.32) & $43.88(11.30)$ & $47.88(10.26)$ & 0.35 \\
\hline \multicolumn{5}{|l|}{ rdIPFC } \\
\hline Glx & 11.66 (1.44) & $11.08(1.55)$ & $11.16(1.65)$ & 0.74 \\
\hline Glx fits (\%SD) & 4.57 (1.69) & $4.80(0.94)$ & $5.19(2.10)$ & 0.36 \\
\hline FWHM & $\begin{array}{c}0.0387 \\
(0.0132)\end{array}$ & $\begin{array}{c}0.0370 \\
(0.0076)\end{array}$ & $\begin{array}{c}0.0389 \\
(0.0091)\end{array}$ & 0.85 \\
\hline SNR & $57.76(12.38)$ & $54.67(12.80)$ & $56.93(13.46)$ & 0.68 \\
\hline
\end{tabular}

661

662 Table S2: Regional concentration means and SDs of GIx and data quality parameters. FWHM: Full-

663 Width Half Maximum; SNR: Signal-to-Noise Ratio. ${ }^{1}$ Kruskal-Wallis $\mathrm{H}$ test 
Glutamate, strong priors and hallucinations

\begin{tabular}{|c|c|c|c|c|c|c|c|c|}
\hline $\begin{array}{r}\text { Models } \\
\text { (free par.) }\end{array}$ & $\begin{array}{c}\text { M1 } \\
\left(\omega_{2}, \omega_{3}, \beta\right)\end{array}$ & $\begin{array}{c}\text { M2 } \\
\left(\omega_{2}, \omega_{3}, \beta, v\right)\end{array}$ & $\begin{array}{c}\text { M3 } \\
\left(\omega_{2}, \beta\right)\end{array}$ & $\begin{array}{c}\text { M4 } \\
\left(\omega_{2}, \beta, v\right)\end{array}$ & $\begin{array}{l}\text { M5 } \\
(\beta)\end{array}$ & $\begin{array}{c}\text { M6 } \\
(\beta, v)\end{array}$ & $\begin{array}{l}\text { M7 } \\
\left(\omega_{2}\right)\end{array}$ & $\begin{array}{c}\text { M8 } \\
\left(\omega_{2}, v\right)\end{array}$ \\
\hline$\mu_{0,2}$ & $0(0)$ & $0(0)$ & $0(0)$ & $0(0)$ & $0(0)$ & $0(0)$ & $0(0)$ & $0(0)$ \\
\hline$\mu_{0,3}$ & $1(0)$ & $1(0)$ & $1(0)$ & $1(0)$ & $1(0)$ & $1(0)$ & $1(0)$ & $1(0)$ \\
\hline$\sigma_{0,2}$ & $0.1(0)$ & $0.1(0)$ & $0.1(0)$ & $0.1(0)$ & $0.1(0)$ & $0.1(0)$ & $0.1(0)$ & $0.1(0)$ \\
\hline$\sigma_{0,3}$ & $1(0)$ & $1(0)$ & $1(0)$ & $1(0)$ & $1(0)$ & $1(0)$ & $1(0)$ & $1(0)$ \\
\hline $\boldsymbol{\kappa}$ & $1(0)$ & $1(0)$ & $1(0)$ & $1(0)$ & $1(0)$ & $1(0)$ & $1(0)$ & $1(0)$ \\
\hline$\omega_{2}$ & $-5.6(16)$ & $-5.6(16)$ & $-5.6(16)$ & $-5.6(16)$ & $-5.6(0)$ & $-5.6(0)$ & $-5.6(16)$ & $-5.6(16)$ \\
\hline$\omega_{3}$ & $-7.5(16)$ & $-7.5(16)$ & $-7.5(0)$ & $-7.5(0)$ & $-7.5(0)$ & $-7.5(0)$ & $-7.5(0)$ & $-7.5(0)$ \\
\hline$\beta$ & $48(2.7)$ & $48(2.7)$ & $48(2.7)$ & $48(2.7)$ & $48(2.7)$ & $48(2.7)$ & $48(0)$ & $48(0)$ \\
\hline$v$ & - & $1(2.7)$ & - & $1(2.7)$ & - & $1(2.7)$ & - & $1(2.7)$ \\
\hline
\end{tabular}

664

665 Table S3: HGF models and sufficient statistics of Gaussian parameter priors. $\mu_{0,2}$ : initial value of $X_{2} ; \mu_{0,3}$ :

666 initial value of $X_{3} ; \sigma_{0,2}$ : initial uncertainty about $X_{2} ; \sigma_{0,3}$ : initial uncertainty about $X_{3} ; \kappa: X_{2}-X_{3}$ coupling;

$667 \omega_{2}$ : stimulus contingency evolution rate; $\omega_{3}$ : volatility evolution rate (metavolatility); $\beta$ : inverse decision

668 temperature; $v$ : prior weighting. In all cases the drift was fixed to 0.

669

670

671

672 